\title{
Anti-SARS-CoV-2 immunogenicity decay and incident cases six months after Sinovac-CoronaVac inactivated vaccine in autoimmune rheumatic diseases patients: phase 4 prospective trial
}

\section{Eloisa Bonfa ( $\nabla$ eloisa.bonfa@hc.fm.usp.br)}

Hospital das Clinicas HCFMUSP, Faculdade de Medicina, Universidade de Sao Paulo https://orcid.org/0000-00020520-4681

\section{Clovis Silva}

Hospital das Clinicas HCFMUSP, Faculdade de Medicina, Universidade de Sao Paulo

Ana Medeiros-Ribeiro

Hospital das Clinicas HCFMUSP, Faculdade de Medicina, Universidade de Sao Paulo

\section{Leonard Kupa}

Hospital das Clinicas HCFMUSP, Faculdade de Medicina, Universidade de Sao Paulo Emily Yuki

Hospital das Clinicas HCFMUSP, Faculdade de Medicina, Universidade de Sao Paulo 8801-405X

\section{Sandra Sandra G. Pasoto}

Hospital das Clinicas HCFMUSP, Faculdade de Medicina, Universidade de Sao Paulo

\section{Carla Saad}

Hospital das Clinicas HCFMUSP, Faculdade de Medicina, Universidade de Sao Paulo

\section{Solange Fusco}

Hospital das Clinicas HCFMUSP, Faculdade de Medicina, Universidade de Sao Paulo

\section{Rosa Pereira}

Hospital das Clinicas HCFMUSP, Faculdade de Medicina, Universidade de Sao Paulo

\section{Samuel Shinjo}

Hospital das Clinicas HCFMUSP, Faculdade de Medicina, Universidade de Sao Paulo

\section{Ari Halpern}

Hospital das Clinicas HCFMUSP, Faculdade de Medicina, Universidade de Sao Paulo

\section{Eduardo Borba}

Hospital das Clinicas HCFMUSP, Faculdade de Medicina, Universidade de Sao Paulo

\section{Fernando Souza}

Hospital das Clinicas HCFMUSP, Faculdade de Medicina, Universidade de Sao Paulo

\section{Lissiane Guedes}

Hospital das Clinicas HCFMUSP, Faculdade de Medicina, Universidade de Sao Paulo

\section{Renata Miossi}

Hospital das Clinicas HCFMUSP, Faculdade de Medicina, Universidade de Sao Paulo

\section{Karina Bonfiglioli}

Hospital das Clinicas HCFMUSP, Faculdade de Medicina, Universidade de Sao Paulo 


\section{Diogo Domiciano}

Hospital das Clinicas HCFMUSP, Faculdade de Medicina, Universidade de Sao Paulo

Andrea Shimabuco

Hospital das Clinicas HCFMUSP, Faculdade de Medicina, Universidade de Sao Paulo

Danieli Andrade

Hospital das Clinicas HCFMUSP, Faculdade de Medicina, Universidade de Sao Paulo

\section{Luciana Seguro}

Hospital das Clinicas HCFMUSP, Faculdade de Medicina, Universidade de Sao Paulo

Ricardo Fuller

Hospital das Clinicas HCFMUSP, Faculdade de Medicina, Universidade de Sao Paulo

\section{Percival Sampaio-Barros}

Hospital das Clinicas HCFMUSP, Faculdade de Medicina, Universidade de Sao Paulo

Ana Paula Assad

Hospital das Clinicas HCFMUSP, Faculdade de Medicina, Universidade de Sao Paulo

\section{Julio Moraes}

Hospital das Clinicas HCFMUSP, Faculdade de Medicina, Universidade de Sao Paulo

\section{Claudia Goldenstein-Schainberg}

Hospital das Clinicas HCFMUSP, Faculdade de Medicina, Universidade de Sao Paulo

Henrique Giardini

Hospital das Clinicas HCFMUSP, Faculdade de Medicina, Universidade de Sao Paulo

\section{Henrique Silva}

Hospital das Clinicas HCFMUSP, Faculdade de Medicina, Universidade de Sao Paulo

Victor Martins

Hospital das Clinicas HCFMUSP, Faculdade de Medicina, Universidade de Sao Paulo

\section{Lorena Villamarin}

Hospital das Clinicas HCFMUSP, Faculdade de Medicina, Universidade de Sao Paulo 8382-1119

\section{Renata Novellino}

Hospital das Clinicas HCFMUSP, Faculdade de Medicina, Universidade de Sao Paulo

\section{Lucas Sales}

Hospital das Clinicas HCFMUSP, Faculdade de Medicina, Universidade de Sao Paulo

\section{Carlo Araújo}

Hospital das Clinicas HCFMUSP, Faculdade de Medicina, Universidade de Sao Paulo

\section{Matheus Silva}

Hospital das Clinicas HCFMUSP, Faculdade de Medicina, Universidade de Sao Paulo

\section{Dilson Filho}

Hospital das Clinicas HCFMUSP, Faculdade de Medicina, Universidade de Sao Paulo

\section{Marta Lopes}

Hospital das Clinicas HCFMUSP, Faculdade de Medicina, Universidade de Sao Paulo

\section{Alberto Duarte}

Hospital das Clinicas HCFMUSP, Faculdade de Medicina, Universidade de Sao Paulo

\section{Alberto Duarte}

Hospital das Clinicas HCFMUSP, Faculdade de Medicina, Universidade de Sao Paulo 
Instituto de Medicina Tropical da Faculdade de Medicina da Universidade de São Paulo, São Paulo

\section{Esper Kallas}

Instituto de Medicina Tropical da Faculdade de Medicina da Universidade de São Paulo, São Paulo

\section{Nadia Aikawa}

Hospital das Clinicas HCFMUSP, Faculdade de Medicina, Universidade de Sao Paulo

\section{Article}

Keywords: anti-SARS-CoV-2 immunogenicity decay, Sinovac-CoronaVac inactivated vaccine, autoimmune rheumatic diseases

Posted Date: November 23rd, 2021

DOI: https://doi.org/10.21203/rs.3.rs-1054476/v1

License: (c) (i) This work is licensed under a Creative Commons Attribution 4.0 International License. Read Full License

Version of Record: A version of this preprint was published at Nature Communications on October 3rd, 2022. See the published version at https://doi.org/10.1038/s41467-022-33042-0. 


\section{Abstract}

We provide novel data on anti-SARS-CoV-2 immunogenicity decay and incident cases six months after the 2nd dose of Sinovac-CoronaVac inactivated vaccine(D210) in 828 autoimmune rheumatic diseases(ARD) patients compared with 223 age/sex-balanced control group(CG). From D69 to D210, anti-S1/S2IgG positivity and GMT reduced $23.8 \%$ and $38 \%$ in $\operatorname{ARD}(p<0.001 / p<0.001)$ and $20 \%$ and $51 \%$ in $C G(p<0.001 / p<0.001)$. From D69 to D210 NAb positivity and activity declined $41 \%$ and $54 \%$ in $\operatorname{ARD}(p<0.001 / p<0.001)$ and $39.7 \%$ and $47 \%$ in $C G(p<0.001 / p<0.001)$. Multivariate logistic regression analysis showed that male(OR=0.56;95\% Cl0.40-0.79; $<<0.001)$, prednisone $(\mathrm{OR}=0.56 ; 95 \% \mathrm{Cl} 0.41$ $0.76 ; p<0.001)$, anti-TNF(OR=0.66;95\%Cl0.45-0.96; $\mathrm{p}=0.031)$, abatacept $(\mathrm{OR}=0.29 ; 95 \% \mathrm{Cl0} .15-0.56 ; \mathrm{p}<0.001)$ and rituximab(OR=0.32;95\% Cl0.11-0.90; $\mathrm{p}=0.031)$ use were associated with a substantial reduction on IgG response at D210 in ARD patients. A decrease of COVID-19 cases(from 27.5 to 8.1/100 person-years; $p<0.001$ ) occurred during the study despite the Delta variant spread. In conclusion, after 6-months of Sinovac-CoronaVac 2nd dose, immunogenicity of ARD patients was markedly reduced, particularly in males and those under prednisone/biological therapies, without a concomitant rise in COVID-19 cases(NCT04754698).

\section{Introduction}

Mass vaccination is the main measure to control the severe acute respiratory syndrome coronavirus 2 (SARS-CoV-2) spread and emergence of new viral variants of concern ${ }^{1}$. While the pandemic drags on, definition of immunogenicity durability is an essential step to establish booster doses strategies.

Data on persistence of post-vaccination immunogenicity are, however, scarce with few cases series on messenger RNA (mRNA) and viral vector vaccines demonstrating in the general population a variable decline of antibody levels in a period of two to six months after two doses of SARS-CoV-2 vaccination ${ }^{2-8}$. In addition, a large prospective study in health care workers reported a substantial decrease of mRNA vaccine induced antibodies in 6-months ${ }^{8}$. Some of these studies identified age and sex as associated with reduced durability of vaccine humoral immune response ${ }^{3,8}$.

The inactivated Sinovac-CoronaVac vaccine is currently used in the most populated countries of the world, and its protective effect against hospitalization and death related to coronavirus infectious disease 2019 (COVID-19) was demonstrated in more than 10 million subjects ${ }^{9}$. The short-term waning of antibody response to this vaccine was evaluated in 159 health care workers with a persistent seropositivity up to 98 days after vaccination, although with significant reduction in levels after 42 days ${ }^{10}$.

The vulnerable population of immunocompromised individuals was also evaluated for durability of vaccine immunogenicity in few cancer patients under active therapy. The follow-up after the second COVID-19 vaccination lasted solely 3-4 months, with a reported decay of IgG titers or inability to sustain IgG levels above the threshold ${ }^{11,12}$. With regard to autoimmune diseases one study assessed 242 patients with a wide range of different conditions using a general computer-based questionnaire. They identified that participants with immunosuppression had a $65 \%$ reduction in IgG levels and $70 \%$ in neutralizing antibody concentrations compared to those without these therapies up to 6 months after mRNA vaccination ${ }^{8}$.

The deleterious impact of immunosuppressive therapy in a large autoimmune rheumatic diseases (ARD) population was reported for primary Sinovac-CoronaVac vaccination in a prospective study ${ }^{13,14}$. However, there is no report evaluating the long-term durability of anti-SARS-CoV-2 immunogenicity in COVID-19 vaccinated ARD patients. 
Here, we described the analysis of a large ARD population, that was conducted to assess prospectively the 6-month durability of SARS-CoV-2 immunogenicity in fully vaccinated adults with Sinovac-CoronaVac compared with age- and sex-balanced control group. We further evaluated incident symptomatic COVID-19 cases confirmed by real-time reverse transcriptase-polymerase chain reaction (RT-PCR). We also assessed risk factors for reduced 6-month durability of anti-SARS-CoV-2 immunogenicity.

\section{Results}

Participants originated from a large single center (Sao Paulo, Brazil) phase 4 controlled prospective study (no. NCT04754698, CoronavRheum) of immunogenicity and safety of two doses of Sinovac-CoronaVac vaccine in ARD patients and controls $(\mathrm{CG})^{13}$. After applying the exclusion criteria, the final study groups consisted of 828 ARD patients and 207 controls vaccinated with 2 doses (Supplementary Figure 1). ARD group included patients with: $27.5 \%(n=228)$ rheumatoid arthritis, $24.9 \%(n=206)$ systemic lupus erythematosus, $23.7 \%(n=196)$ axial spondyloarthritis, $6.2 \%(n=51)$ primary vasculitis, $4.8 \%(n=40)$ idiopathic inflammatory myopathies, $4.3 \%(n=36)$ systemic sclerosis, and $4.3 \%(n=36)$ primary Sjögren's syndrome. Regarding ARD current therapy at 6 weeks after the $2^{\text {nd }}$ dose (D69) the most frequently used were: $62.0 \%(n=513)$ immunosuppressive drugs, 36.5\% ( $\left.n=302\right)$ prednisone and $35.7 \%(n=296)$ biologic therapy. ARD and CG groups were comparable regarding median current age $(p=0.898)$, female sex $(p>0.999)$ and Caucasian race $(p=0.163)$ (Supplementary Table 1$)$.

Anti-SARS-CoV-2 S1/S2 IgG assessed from D69 (6 weeks after vaccine second dose) to D210 are presented in Table 1. From D69 to D210, anti-S1/S2 IgG seropositivity rates reduced 23.8\% in ARD [650 (78.5\%) vs. 495 (59.8\%), p < 0.001] and 20\% in CG [202 (97.6\%) vs. 161 (77.8\%), p<0.001], with moderate but lower IgG persistence in ARD compared to CG at D210 ( $<<0.001)$. IgG GMT from D69 to D210 declined significantly after the second dose in ARD [41.8 (38.0-46.0) vs. 26.1 (23.2-29.4) AU/mL, p<0.001] and in CG [99.6 (88.2-112.6) vs. 48.8 (40.3-59.0) AU/mL, $\mathrm{p}<0.001]$, with lower IgG levels in the former group at D210 ( $<<0.001)$ (Table 1). The decrease in IgG titer, calculated as 1 - Ln(lgG210/lgGD69), was significantly lower in ARD compared to CG [38\% (95\%Cl 32-43\%) vs. 51\% (95\% Cl 43$58 \%), p=0.004]$.

From D69 to D210, NAb positivity declined 41\% in ARD [539 (65.1\%) vs. 318 (38.4\%), p<0.001] and $39.7 \%$ in CG [181 (87.4\%) vs. 109 (52.7\%), p<0.001]. In the same period, a substantial reduction of $41 \%$ in NAb activity was observed in ARD [47.7\% (IQR 15.0-75.1) vs. 15\% (IQR 15.0-52.0), p<0.001] and of 39.7\% in CG [66.1\% (IQR 45.2-91.0) vs. 34.5\% (IQR 15-76.8), $\mathrm{p}<0.001]$. The decrease in NAb activity [1 - Ln(NAb activityD210/NAb activityD69)] in positive individuals at D69 was significantly higher in ARD compared to CG [54\% (95\% Cl 51-57\%) vs. 47\% (95\% Cl 41-52\%); $\mathrm{p}=0.024]$.

At D210, negative anti-S1/S2 IgG in ARD group was associated with older age $(p=0.001)$, lower frequencies of systemic lupus erythematosus $(p=0.005)$ and systemic sclerosis $(p=0.024)$ and higher frequency of male sex $(p=0.007)$ and rheumatoid arthritis $(p<0.001)$. Regarding the influence of current therapy, patients seronegative for IgG at D210 were more often under prednisone (43.7\% vs. 31.1\%, $\mathrm{p}<0.001)$, anti-TNF $(19.2 \%$ vs. $13.2 \%, \mathrm{p}=0.012)$, abatacept $(9.8 \%$ vs. $2.7 \%, \mathrm{p}<0.001)$ and rituximab (3\% vs. $1.1 \%, \mathrm{p}=0.028)$, while hydroxychloroquine use was more frequent in ARD with positive anti-S1/S2 IgG (Table 2). Multivariate logistic regression analysis using IgG positivity at D210 as the dependent variable and as independent variables those with $p<0.2$ in univariate analysis, revealed that male sex $(\mathrm{OR}=0.56,95 \% \mathrm{Cl} 0.40-0.79, \mathrm{p}<0.001)$, prednisone use $(\mathrm{OR}=0.56 ; 95 \% \mathrm{Cl} 0.41-0.76, \mathrm{p}<0.001)$, anti-TNF use $(\mathrm{OR}=0.66 ; 95 \% \mathrm{Cl} 0.45-0.96, \mathrm{p}=0.031)$, abatacept use $(\mathrm{OR}=0.29 ; 95 \% \mathrm{Cl} 0.15-0.56, \mathrm{p}<0.001)$ and rituximab use $(\mathrm{OR}=0.32 ; 95 \% \mathrm{Cl} 0.11-0.90, \mathrm{p}=0.031)$ were significantly associated with absence of anti-S1/S2 IgG six months after vaccination in ARD patients. 
For NAb analysis at D210, SLE diagnosis was less frequent in seronegative ARD patients $(p=0.019)$ whereas biologic therapy $(p=0.031)$, particularly abatacept use $(p=0.018)$ was higher in patients without NAb (Table 2). After multivariate logistic regression analysis using NAb positivity as the dependent variable and as independent variables those with $p<0.2$ in univariate analysis, only abatacept use $(\mathrm{OR}=0.24 ; 95 \% \mathrm{Cl} 0.13-0.46, p=0.041)$ remained significantly associated with absence of NAb at D210 in ARD patients.

Analysis of incident cases of RT-PCR confirmed COVID-19 were evaluated from first dose to 10 days after the second dose (T1) and thereafter to 180 days after the second dose (T2). Evaluation of incident cases among ARD patients $(n=1193)$ revealed $n=79$ cases $(n=33$ in T1 and $n=46$ in T2) of COVID-19 with $n=14$ hospitalizations ( $n=7$ in T1 and $n=7$ in T2) and $n=4$ deaths ( $n=1$ in T1 and $n=3$ in T2). The incident symptomatic COVID-19 cases reduced from 27.5 (95\% Cl 18.9-38.6)/100 person-years in $\mathrm{T} 1$ to 8.1 (95\% $\mathrm{Cl} 6.0-10.9) / 100$ person-years in $\mathrm{T} 2$, with an incidence rate decline of $19.4(95 \% \mathrm{Cl} 12.7-26.1)$ cases/100 person-years $(\mathrm{p}<0.001)$. Likewise, in the same time periods hospitalizations due to COVID-19 decreased from $5.8(95 \% \mathrm{Cl} 2.3-12.0) / 100$ person-years to $1.2(95 \% \mathrm{Cl} 0.5-2.6)-/ 100$ person-years, with an incidence rate decline of $4.6(95 \% \mathrm{Cl} 1.8-7.4)$ hospitalizations/100 person-years $(p=0.001)$.

Deaths due to COVID-19 occurred in n=4 ARD patients: $n=1$ in T1 and $n=3$ in T2 ( $p=0.694)$, after the $2^{\text {nd }}$ dose of Sinovac-CoronaVac. The first death occurred in a 58 years-old female rheumatoid arthritis patient with bronchiolitis, diabetes mellitus, arterial hypertension, obesity, and hypothyroidism, under rituximab (last dose 5 months earlier) and prednisone $2.5 \mathrm{mg} /$ day. The second death was on a 72 years-old male rheumatoid arthritis patient with chronic renal failure, pleural effusion, asthma, and dyslipidemia, under hydroxychloroquine and prednisone $2.5 \mathrm{mg} /$ day. Third death occurred in a 73 years-old female, with idiopathic inflammatory myopathy and interstitial lung disease, arterial hypertension, diabetes mellitus, and dyslipidemia, under mycophenolate mofetil, and another in a 65 years-old female patient, with granulomatosis with polyangiitis and arterial hypertension, under azathioprine and prednisone (2.5 $\mathrm{mg} /$ day).

Further comparative survival analysis of ARD and CG $(p=0.152)$, with random selection of age and sex comparable subjects pointed that $n=33$ (ARD $n=31$ and CG $n=2$ ) RT-PCR confirmed COVID-19 incident symptomatic cases were reported during T1 (40 days) and $n=52$ cases (ARD $n=44$ in CG $n=8$ ) during T2 (180 days) evenly distributed along this period in ARD patients (Supplementary Figure 2).

\section{Discussion}

To our knowledge this is the first study to specifically demonstrate a substantial decline of anti-SARS-CoV-2 immunogenicity in ARD patients 6-months after the full inactivated vaccine schedule without a simultaneous increase in breakthrough cases. We further identified male sex and immunosuppression as deleterious for long-term antibody persistence in these patients.

The large number of ARD patients with a balanced age and sex CG included in this study was a main strength since it provided a unique opportunity to define more accurately risk factors for vaccine induced anti-SARS-CoV-2 durability. In fact, age and sex were identified previously as determinants of inability to sustain SARS-CoV-2 antibody levels in health population ${ }^{3}$. The use of established classification criteria for each disease allowed a more accurate definition of the impact of different conditions and their therapy. The inclusion of a broad spectrum of non-rheumatologic immunosuppressed participants and the use of a generic computer-based questionnaire about existing conditions and treatment precluded a definitive conclusion about the subgroup of ARD and their treatment in a previous study ${ }^{8}$. 
The period of 6 months with a parallel observation of incident cases is endorsed by the report of breakthrough infection 4-month post-BTN162b2 vaccination associated with reduced levels of antibodies peri-infection and a significantly diminished humoral response in 6-months ${ }^{1,8}$. The uniform post-vaccination follow-up was an essential parameter for a more accurate definition of vaccine induced antibodies persistence at 6 months. In fact, previous studies have demonstrated that vaccine humoral response waning dynamics varies overtime and is also distinct for IgG and NAb level ${ }^{8}$. An important limitation of the present study is the non-assessment of cellular immunity in this population, but neutralizing antibodies evaluated herein were reported to be associated with protective immunity ${ }^{15}$.

We provide novel data of a reduction of anti-SARS-CoV-2 antibodies positivity over 6-months post Sinovac-CoronaVac second dose in ARD patients with a magnitude of approximately $20 \%$ for patients and controls. We also confirmed the observation of a robust ( $\geq 4$ times) decrease in SARS-CoV-2 mRNA vaccine IgG levels after 6-months in health care workers $^{8}$. We further demonstrated that the same phenomena, although less intense, occurred for the SinovacCoronoVac with a more expressive decrease in controls (51\%) than in ARD patients (38\%) at 6 months post second dose.

The analysis of NAb activity, reported to be a strong correlate of protection ${ }^{15}$, revealed a substantial waning of $54 \%$ after 6-months of Sinovac-CoronaVac vaccination in ARD patients. A similar finding of $70 \%$ reduction in NAb titer 6 months post-mRNA vaccination was reported for participants with immunosuppression ${ }^{8}$. In contrast, a less prominent decline in NAb positivity of approximately $40 \%$ for ARD patients and controls was found herein.

Regarding deleterious factors for vaccine-induced immunogenicity durability, we have identified in the multivariate logistic regression analysis that male sex, prednisone and anti-TNF have a moderate impact in immunogenicity persistence whereas a major harmful effect was evidenced for abatacept and rituximab after 6-months. For NAb positivity, only abatacept was associated with absence of these antibodies at D210 in ARD patients. Likewise, a significant lower NAb activity level was reported in men and immunosuppressed health worker participants 6-months after receipt of the second dose ${ }^{8}$.

The predominance of incident cases after the vaccine first shot up to 10 days after second dose (T1) contrast with the significant drop in infection and hospitalization in the study participants in the subsequent 40 days which coincided with the second peak of COVID-19 cases in São Paulo city (45\% increase in the same time period) ${ }^{16}$. Interestingly, the emerging of Delta (B.1.617.2) variant in Sao Paulo in July ${ }^{17}$ with a rapid spread in the following months did not lead to a parallel upsurge of COVID-19 breakthrough cases among our cohort which remained with homogeneous distribution of cases. Our data supports the notion that Sinovac-CoronaVac may sustain a 6-month effectiveness against Delta strain.

In conclusion, we provide novel data on the long-term Sinovac-CoronaVac immunogenicity in ARD patients demonstrating a significant decrease in IgG and NAb levels 6 months after the second dose without a corresponding rise in symptomatic COVID-19 cases in the same period. Male, prednisone and biological therapy were identified as the main contributing factors to reduced durability of vaccine induced humoral response.

\section{References}

1. Bergwerk, M. et al. Covid-19 Breakthrough Infections in Vaccinated Health Care Workers. N. Engl. J. Med. 385 , 1474-1484 (2021).

2. McDade, T. W. et al. Durability of antibody response to vaccination and surrogate neutralization of emerging variants based on SARS-CoV-2 exposure history. Sci. Rep. 11, 17325 (2021).

Page $7 / 11$ 
3. Widge, A. T. et al. Durability of Responses after SARS-CoV-2 mRNA-1273 Vaccination. N. Engl. J. Med. 384, 80-82 (2021).

4. Shrotri, M. et al. Spike-antibody waning after second dose of BNT162b2 or ChAdOx1. Lancet 398, 385-387 (2021).

5. Barouch, D. H. et al. Durable Humoral and Cellular Immune Responses 8 Months after Ad26.COV2.S Vaccination. N. Engl. J. Med. 385, 951-953 (2021).

6. Doria-Rose, N. et al. Antibody Persistence through 6 Months after the Second Dose of mRNA-1273 Vaccine for Covid-19. N. Engl. J. Med. 384, 2259-2261 (2021).

7. Pegu, A. et al. Durability of mRNA-1273-induced antibodies against SARS-CoV-2 variants. Preprint at https://www.biorxiv.org/content/10.1101/2021.05.13.444010v1 (2021).

8. Levin, E. G. et al. Waning Immune Humoral Response to BNT162b2 Covid-19 Vaccine over 6 Months. N. Engl. J. Med. https://www.nejm.org/doi/10.1056/NEJMoa2114583 (2021).

9. Jara, A. et al. Effectiveness of an Inactivated SARS-CoV-2 Vaccine in Chile. N. Engl. J. Med. 385, 875-884 (2021).

10. Cucunawangsih, C., Wijaya, R. S., Lugito, N. P. H. \& Suriapranata, I. Antibody response to the inactivated SARSCoV-2 vaccine among healthcare workers, Indonesia. Int. J. Infect. Dis. https://www.sciencedirect.com/science/article/pii/S1201971221007876?via\%3Dihub (2021).

11. Eliakim-Raz, N., Massarweh, A., Stemmer, A. \& Stemmer, S. M. Durability of Response to SARS-CoV-2 BNT162b2 Vaccination in Patients on Active Anticancer Treatment. JAMA Oncol. https://jamanetwork.com/journals/jamaoncology/fullarticle/2783031 (2021).

12. Tadmor, T. et al. Antibody persistence 100 days following the second dose of BNT162b mRNA Covid19 vaccine in patients with chronic lymphocytic leukemia. Leukemia 2021 35, 2727-2730 (2021).

13. Medeiros-Ribeiro, A. C. et al. Immunogenicity and safety of the CoronaVac inactivated vaccine in patients with autoimmune rheumatic diseases: a phase 4 trial. Nat. Med. 27, 1744-1751 (2021).

14. Aikawa, N. E. et al. Phase 4 prospective analysis of immunogenicity and safety of two doses of SinovacCoronaVac COVID-19 vaccine in SARS-CoV-2-exposed and nal̈ve autoimmune rheumatic disease patients. Lancet. Rheumatol. In press (2021).

15. Khoury, D. S. et al. Neutralizing antibody levels are highly predictive of immune protection from symptomatic SARS-CoV-2 infection. Nat. Med. 27, 1205-1211 (2021).

16. Coronavírus - Boletim Completo. Governo do Estado de São Paulo https://www.seade.gov.br/coronavirus/\# (2020).

17. Lamarca, A. P. et al. Genomic surveillance tracks the first communitary outbreak of Delta (B.1.617.2) variant in Brazil https://virological.org/t/genomic-surveillance-tracks-the-first-communitary-outbreak-of-delta-b-1-617-2variant-in-brazil/733

\section{Tables}

Table 1 - Anti-SARS-CoV-2 S1/S2 IgG seropositivity (SP) rates and titers six weeks (D69) and 6 months (D210) after second dose of Sinovac-CoronaVac vaccination in patients with $A R D$ in comparison to CG 


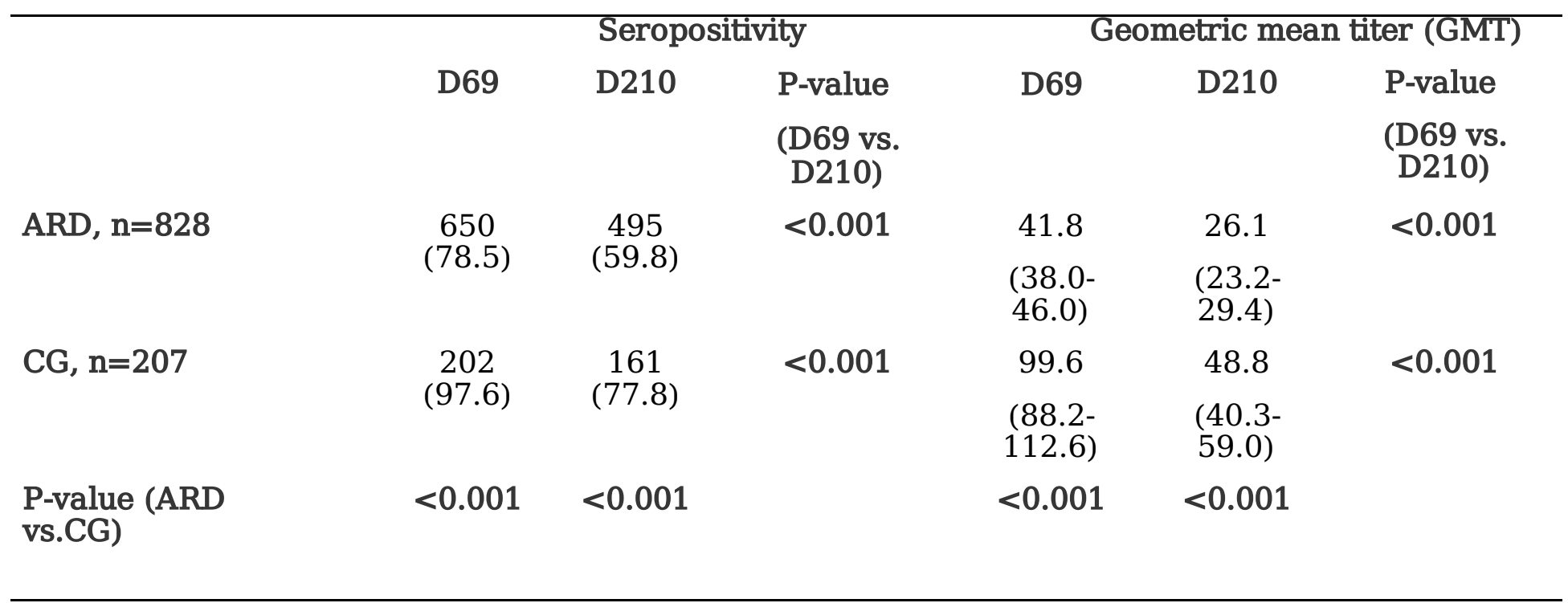

ARD - autoimmune rheumatic diseases; CG - control group

Seropositivity was defined as post vaccination titer $\geq 15 \mathrm{AU} / \mathrm{mL}$ - Indirect ELISA (LIAISON ${ }^{\circledR}$ SARSCoV-2 S1/S2 IgG, DiaSorin, Italy).

GMT - Geometric mean titers (AU/mL);

Frequencies of seropositivity are presented as number (\%) and they were compared between groups (ARD and CG) and between time points (D69 vs. D210) using generalized estimating equations (GEE) with binomial distribution and logit link function, assuming autoregressive correlation matrix between moments.

IgG antibody titers are expressed as geometric means with 95\% confidence interval (95\%CI). Data regarding IgG titers were analyzed at neperian logarithm (ln)-transformed basis using Serology parameters were compared between groups (ARD and CG) and timepoints (D69 and D210) using GEE with normal marginal distribution and gamma distribution respectively and identity binding function assuming first order autoregressive correlation matrix between moments.

Results were followed by Bonferroni multiple comparisons to identify differences between groups and timepoints.

All analyses were two-sided.

Sharacteristics of autoimmune rheumatic diseases (ARD) patients six weeks after the $2^{\text {nd }}$ dose (D69) $\mathrm{w}$ positivity (SP) for anti-SARS-CoV-2 S1/S2 IgG antibodies and without and with neutralizing antibodie: fter second dose of Sinovac-CoronaVac vaccination (D210) 


\begin{tabular}{|c|c|c|c|c|c|c|}
\hline & $\begin{array}{l}\text { Patients without SP } \\
\text { (S1/S2 IgG) } \\
(n=396)\end{array}$ & $\begin{array}{c}\text { Patients } \\
\text { with } \\
\text { SP (S1/S2 } \\
\text { IgG) } \\
(n=560)\end{array}$ & $p$ value & $\begin{array}{c}\text { Patients } \\
\text { without NAb } \\
(n=599)\end{array}$ & $\begin{array}{c}\text { Patients with } \\
\text { NAb } \\
(n=357)\end{array}$ & \\
\hline \multicolumn{7}{|l|}{ p̣hic data } \\
\hline age, years & $53(42-63)$ & $50(39-60)$ & 0.001 & $52(40-62)$ & $51(40-60)$ & 0.382 \\
\hline age 60 years & $128(32.3)$ & $153(27.3)$ & 0.094 & $182(30.4)$ & 99 (27.7) & 0.384 \\
\hline 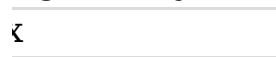 & $115(29.0)$ & $116(20.7)$ & 0.007 & $155(25.9)$ & $76(21.3)$ & 0.219 \\
\hline \multirow[t]{5}{*}{ æn race } & $214(54.0)$ & $281(50.2)$ & 0.239 & 309 (51.6) & $186(52.1)$ & 0.878 \\
\hline & $140(35.4)$ & $136(24.3)$ & $<0.001$ & $184(30.7)$ & $92(25.8)$ & 0.102 \\
\hline & $52(13.1)$ & $69(12.3)$ & 0.711 & $81(13.5)$ & $40(11.2)$ & 0.297 \\
\hline & $41(10.4)$ & $55(9.8)$ & 0.787 & $63(10.5)$ & $33(9.2)$ & 0.526 \\
\hline & $80(20.2)$ & $158(28.2)$ & 0.005 & $134(22.4)$ & $104(29.1)$ & 0.019 \\
\hline \multirow[t]{5}{*}{ vasculitis } & $27(6.8)$ & $34(6.1)$ & 0.642 & $39(6.5)$ & $22(6.2)$ & 0.818 \\
\hline & $18(4.5)$ & $25(4.5)$ & 0.952 & $23(3.8)$ & $20(5.6)$ & 0.203 \\
\hline & $10(2.5)$ & $31(5.5)$ & 0.024 & $23(3.8)$ & $18(5.0)$ & 0.375 \\
\hline & $12(3.0)$ & $29(5.2)$ & 0.106 & $25(4.2)$ & $16(4.5)$ & 0.820 \\
\hline & $16(4.0)$ & $23(4.1)$ & 0.959 & $27(4.5)$ & $12(3.4)$ & 0.715 \\
\hline \multicolumn{7}{|l|}{ herapies } \\
\hline chloroquine & $93(23.5)$ & $185(33.0)$ & 0.001 & 167 (27.9) & $111(31.1)$ & 0.290 \\
\hline azine & $42(10.6)$ & $44(7.9)$ & 0.143 & $63(10.5)$ & $23(6.4)$ & 0.033 \\
\hline me & $173(43.7)$ & $174(31.1)$ & $<0.001$ & $225(37.6)$ & $122(34.2)$ & 0.292 \\
\hline uppressive & $255(64.4)$ & $338(60.4)$ & 0.205 & $368(61.4)$ & $225(63.0)$ & 0.624 \\
\hline trexate & $105(26.5)$ & $144(25.7)$ & 0.781 & $153(25.5)$ & $96(26.9)$ & 0.646 \\
\hline omide & $62(15.7)$ & $71(12.7)$ & 0.190 & $86(14.4)$ & $47(13.2)$ & 0.606 \\
\hline henolate & $53(13.4)$ & $72(12.9)$ & 0.812 & $73(12.2)$ & $52(14.6)$ & 0.291 \\
\hline oprine & $38(9.6)$ & $63(11.2)$ & 0.412 & $60(10.0)$ & $41(11.5)$ & 0.475 \\
\hline inib & $9(2.3)$ & $14(2.5)$ & 0.821 & $14(2.3)$ & $9(2.5)$ & 0.858 \\
\hline \multirow{2}{*}{$\begin{array}{l}\text { phosphamide } \\
\text { ic agent }\end{array}$} & $1(0.3)$ & $6(1.1)$ & 0.250 & $5(0.8)$ & $2(0.6)$ & 0.630 \\
\hline & $179(45.2)$ & 165 (29.5) & $<0.001$ & $231(38.6)$ & $113(31.7)$ & 0.031 \\
\hline TNF & $76(19.2)$ & $74(13.2)$ & 0.012 & $102(17.1)$ & $48(13.4)$ & 0.141 \\
\hline cept & $39(9.8)$ & $15(2.7)$ & $<0.001$ & $42(7.0)$ & $12(3.4)$ & 0.018 \\
\hline zumab & $20(5.1)$ & $32(5.7)$ & 0.656 & $28(4.7)$ & $24(6.7)$ & 0.177 \\
\hline umab & $16(4.0)$ & $15(2.7)$ & 0.242 & $19(3.2)$ & $12(3.4)$ & 0.873 \\
\hline mab & $12(3.0)$ & $6(1.1)$ & 0.028 & $14(2.3)$ & $4(1.1)$ & 0.181 \\
\hline
\end{tabular}

e expressed in mean \pm standard deviation, median (interquartile range) and $\mathrm{n}(\%)$.

imatoid arthritis; SpA - spondyloarthritis; PA - psoriatic arthritis; SLE - systemic lupus erythematosus inflammatory myopathy; SSc - systemic sclerosis; SS - Sjögren's syndrome; PAPS - primary antiphosr

positivity (IgG titer $\geq 15 \mathrm{AU} / \mathrm{ml}$ ) for anti-SARS-CoV-2 S1/S2 IgG antibodies after vaccination (Indirect ] 3) SARS-CoV-2 S1/S2 IgG, DiaSorin, Italy). Positivity for NAbs was defined as a neutralizing activity $\geq$ NT Kit, GenScript, Piscataway, USA).

\section{Supplementary Files}

This is a list of supplementary files associated with this preprint. Click to download. 
- MethodsantibodydecayfinalDoubleBlind.docx

- SupplementaryFigure1.docx

- SupplementaryFigure25.11.21.pdf

- SupplementaryTable1.docx 